intersecting the sphere $x^{2}+y^{2}+z^{2}=1$ in confocal spheroconics; then the differential equations

$$
\begin{aligned}
& \frac{d \mu}{\sqrt{ }\left\{\left(\mu^{2}-b^{2}\right)\left(\mu^{2}-a^{2}\right)\right\}} \pm \frac{d \nu}{\sqrt{ }\left\{\left(b^{2}-\nu^{2}\right)\left(a^{2}-\nu^{2}\right)\right\}}=0, \\
& \frac{d \mu}{\sqrt{ }\left\{\left(c^{2}-\mu^{2}\right)\left(a^{2}-\mu^{2}\right)\right\}} \pm \frac{d \nu}{\sqrt{ }\left\{\left(c^{2}-\nu^{2}\right)\left(a^{2}-\nu^{2}\right)\right\}}=0 \text {, } \\
& \frac{\mu d \mu}{\sqrt{ }\left\{\left(\mu^{2}-b^{2}\right)\left(c^{2}-\mu^{2}\right)\left(\mu^{2}-a^{2}\right)\right\}} \pm \frac{\nu d \nu}{\sqrt{ }\left\{\left(b^{2}-v^{2}\right)\left(c^{2}-\nu^{2}\right)\left(a^{2}-\nu^{2}\right)\right\}}=0 \text {, }
\end{aligned}
$$

represent the three systems of circles having double contact with the sphero-conic $\mu=\alpha$, and these it is easy to see from geometrical considerations are real. Circles of course may be considered as conics having double contact with $\mu=\infty$, or $x^{2}+y^{2}+z^{2}=0$. By projection then on a plane it would appear that we could have three real systems of conics having double contact with one real conic and one imaginary conic.

It would be interesting also to consider the reality of the conics having double contact with three quadrics. For instance, it does not seem possible to have any real conics having real double contact with three confocal ellipsoids. But I must defer the consideration of this question to another opportunity.

\title{
ASYMPTOTIC LINES ON A CIRCULAR RING.*
}

BY PROFESSOR H. MASCHKE.

THE equation of the asymptotic lines of a tore as well as the expression of the length of the arc of these curves leads to elliptic functions. The formulas appear in a remarkably simple form. $\dagger$

Assuming the radius $C A$ of the rotating circle $=1$, and denoting the distance $C O$ of its centre from the axis of rotation by $\lambda$, the tore is represented by these equations:

$$
x=v \cos u, \quad y=v \sin u, \quad z=\sqrt{1-(v-\lambda)^{2}},
$$

* Read before the American Mathematicar Socierty at its Secon d Summer Meeting, Springfield, Mass., August 28, 1895.

+ I have not found any literature with regard to this subject excepting a short notice in the Fortschritte der Mathematik, 1889, page 765, where reference is made to T. Motoda, "Asymptotic lines on a circular ring," Tokio Math. Soc., vol. 4, 217-219, a paper which has been inaccessible to me. 
where $u$ is the angle between the plane of the revolving circle and the $X Z$-plane and $v$ the distance of a point $P$ of the surface from the $Z$-axis, the axis of rotation.

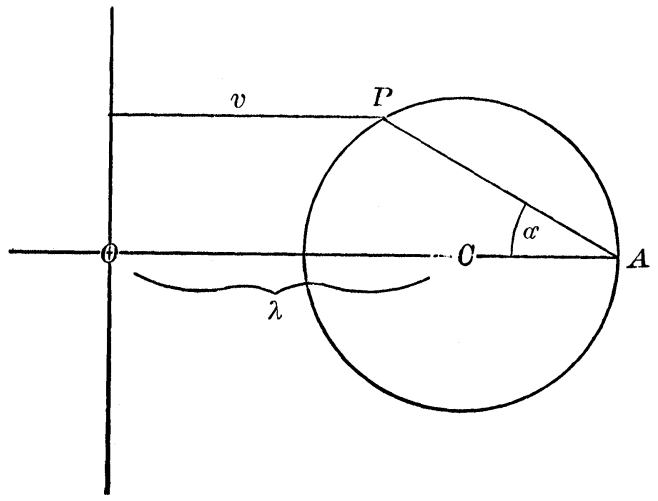

Then the differential equation of the asymptotic curves is this:

$$
\left(\frac{d v}{d u}\right)^{2}=v(\lambda-v)(1+\lambda-v)(1-\lambda+v) .
$$

From this equation we find $v$ in terms of Weierstrass's $8-$ function

$$
v=\frac{2 \lambda(6 \wp u-1)}{12 \curlyvee u+1}
$$

the invariants of $\wp u$ being

and

$$
g_{2}=\frac{1+3 \lambda^{2}}{12}, \quad g_{3}=\frac{1-9 \lambda^{2}}{12.18}
$$

$$
e_{1}=\frac{3 \lambda-1}{12}, \quad e_{2}=\frac{1}{6}, \quad e_{3}=-\frac{3 \lambda+1}{12}
$$

In terms of $\sigma$-functions there is:

$$
v=\frac{2 \lambda \sigma_{2}^{2} u}{\sigma_{1}^{2} u+\sigma_{3}^{2} u}
$$

and denoting $\Varangle O A P=\alpha$,

$$
\tan \alpha=\frac{\sigma_{1} u}{\sigma_{\mathrm{s}} u}
$$


The length of the arc of an asymptotic curve is given by the integral

$$
s=\sqrt{\lambda} \int_{v}^{\lambda} \frac{d v}{\sqrt{(\lambda-v)(1+\lambda-v)(1-\lambda+v)}} .
$$

Introducing the 8 -function with the invariants $g_{2}=\frac{1}{4 \lambda^{2}}$, $g_{3}=0$, and $e_{1}=\frac{1}{4 \lambda}, e_{2}=0, e_{8}=-\frac{1}{4 \lambda},\left(k^{2}=\frac{e_{2}-e_{3}}{e_{1}-e_{3}}=\frac{1}{2}\right)$, we obtain:

$$
\lambda-v=\frac{1}{4 \lambda} \cdot \frac{1}{\wp s} .
$$

University of Chicago, August 23, 1895.

\section{ON A GENERALIZATION OF WEIERSTRASS'S EQUATION WITH THREE TERMS. \\ BY PROFESSOR F._MORLEY.}

THE expression

$$
\prod_{\lambda=1}^{n} \sigma\left(u-b_{\lambda}\right) / \sigma\left(u-a_{\lambda}\right)
$$

is an elliptic function of $u$ if

$$
\Sigma a_{\lambda}=\Sigma b_{\lambda} .
$$

The sum of the residues is zero; that is,

$$
\sum_{\lambda=1}^{n} \frac{\sigma\left(a_{\lambda}-b_{1}\right) \ldots \sigma\left(a_{\lambda}-b_{\lambda}\right) \ldots \sigma\left(a_{\lambda}-b_{n}\right)}{\sigma\left(a_{\lambda}-a_{1}\right) \ldots \quad 1 \quad \ldots \sigma\left(a_{\lambda}-a_{n}\right)}=0 .
$$

Being now only concerned with differences, we can, by a suitable addition to each $a$ and $b$, write

$$
\Sigma a_{\lambda}=\Sigma b_{\lambda}=0 \text {. }
$$

When $n=2$, the equation (1) is in no way characteristic of the $\sigma$-function, but is true of any odd function.

When $n=3$, (1) becomes

$$
\begin{aligned}
& \sigma\left(a_{1}-b_{1}\right) \sigma\left(a_{1}-b_{2}\right) \sigma\left(a_{1}-b_{8}\right) \sigma\left(a_{2}-a_{3}\right) \\
+ & \sigma\left(a_{2}-b_{1}\right) \sigma\left(a_{2}-b_{2}\right) \sigma\left(a_{2}-b_{3}\right) \sigma\left(a_{3}-a_{1}\right) \\
+ & \sigma\left(a_{3}-b_{1}\right) \sigma\left(a_{3}-b_{2}\right) \sigma\left(a_{3}-b_{3}^{3}\right) \sigma\left(a_{1}-a_{3}\right)=0 .
\end{aligned}
$$

